\title{
Anterior Eye Profilometry-guided Scleral Contact Lens Fitting in Keratoconus
}

\author{
${ }^{1}$ David P Piñero, ${ }^{2}$ Roberto Soto-Negro
}

\begin{abstract}
We report the case of a 35-year-old woman diagnosed with keratoconus since she was 18 years old and wearer of corneal rigid contact lenses (CLs). We refitted the case with the fully scleral CL ICD16.5 (Paragon Vision Sciences) for obtaining not only a successful visual restoration, but also a comfortable wear. We initiated the fitting with the spherical model of the CL, but it failed due to instability of the lens. We confirmed the presence of a clear asymmetry in the anterior scleral geometry in both eyes by using the profilometer eye surface profiler (ESP, Eaglet Eye), with a difference between nasal and temporal sagittal heights of 470 and $170 \mu \mathrm{m}$ in right and left eyes respectively. Although this profile suggested the need for the fitting of a CL with significant peripheral toricity, we followed the manufacturer's guidelines and performed a trial with a CL of moderate peripheral toricity $(125 \mu \mathrm{m}$ of difference between steep and flat meridian). The stability of the CL failed again and finally a CL with a peripheral toricity close to that measured with the profilometer was fitted. With this lens, good visual performance, lens stability, and comfort was obtained and maintained during a 1-year follow-up. This case suggests that fully scleral CLs fitting might be optimized with the use of corneo-scleral profilometers, minimizing potentially the number of trials. This potential benefit should be investigated further in future studies.
\end{abstract}

Keywords: Contact lenses, Gas-permeable contact lenses, Keratoconus, Sclera.

How to cite this article: Piñero DP, Soto-Negro R. Anterior Eye Profilometry-guided Scleral Contact Lens Fitting in Keratoconus. Int J Kerat Ect Cor Dis 2017;6(2):97-100.

\section{Source of support: Nil}

Conflict of interest: None

\section{INTRODUCTION}

Scleral CLs have become a common option in clinical practice for the management of irregular cornea, including corneal ectatic conditions. ${ }^{1,2}$ Initially, the fitting of this type of $\mathrm{CL}$ was considered as a challenging issue as there was no device providing a clinical characterization

\footnotetext{
${ }^{1}$ Researcher and Lecturer, ${ }^{2}$ Optometrist

${ }^{1}$ Department of Optics, Pharmacology and Anatomy, University of Alicante, Alicante, Spain

${ }^{2}$ Department of Ophthalmology, Vithas Medimar International Hospital, Alicante, Spain

Corresponding Author: David P Piñero, Researcher and Lecturer, Department of Optics, Pharmacology and Anatomy University of Alicante, Alicante, Spain, Phone: +34965903500 e-mail: david.pinyero@ua.es
}

of the sclero-conjunctival area, where the lens rests. ${ }^{3}$ In the last years, the fitting of this type of CL has been simplified significantly with the introduction of anterior segment optical coherence tomography (OCT). This technology allows the practitioner to achieve significantly better control of the fitting, especially of the lens vault. ${ }^{4}$ However, the selection of the design of the peripheral area of the lens is still performed according to empirical trials in which the centration and peripheral bearing of the lens are evaluated on slit-lamp examination or OCT. This may require some trials that may be tedious for both patient and practitioner. It should be considered that the anterior sclera is not symmetrical and its shape is person dependent. ${ }^{5}$ A new tool has been recently developed to evaluate with accuracy the geometric profile of cornea and anterior sclera, the profilometer. ${ }^{6}$ This technology has been suggested to be potentially useful for scleral CL fitting, but there are no reports to this date showing the potential applicability of this type of devices in this field. The aim of the current case report was to show the applicability of a corneo-scleral profilometer for guiding the fitting of a fully scleral CL in two keratoconus corneas with asymmetrical sclero-conjunctival profile.

\section{CASE REPORT}

We report the case of a 35-year-old woman diagnosed with keratoconus since she was 18 years old. She wore, in the last 2 years, corneal rigid gas-permeable CL, but with discomfort and high levels of intolerance. On pre-refitting examination, refraction was $-5.25-3.50 \times 80^{\circ}$ and -7.00 $-5.00 \times 120^{\circ}$ in right (RE) and left eyes (LE) respectively. Corrected distance visual acuity (CDVA) was 0.00 and 0.10 $\log$ MAR in RE and LE respectively. An analysis of the cornea with the Scheimpflug imaging-based topography system Sirius (CSO, Firenze, Italy) revealed the presence of a significant corneal asymmetry and a moderate corneal thinning in both eyes (Fig. 1).

We decided to refit the case with fully scleral CL for obtaining not only a successful visual restoration, but also a comfortable wear. These lenses do not have corneal contact and, therefore, only rest on the sclero-conjunctival surface. Specifically, the CL ICD16.5 (irregular corneal design) was fitted (Paragon Vision Sciences, distributed by Lenticon, Madrid, Spain) that has four differentiated zones, allowing a correct centration with no corneal 


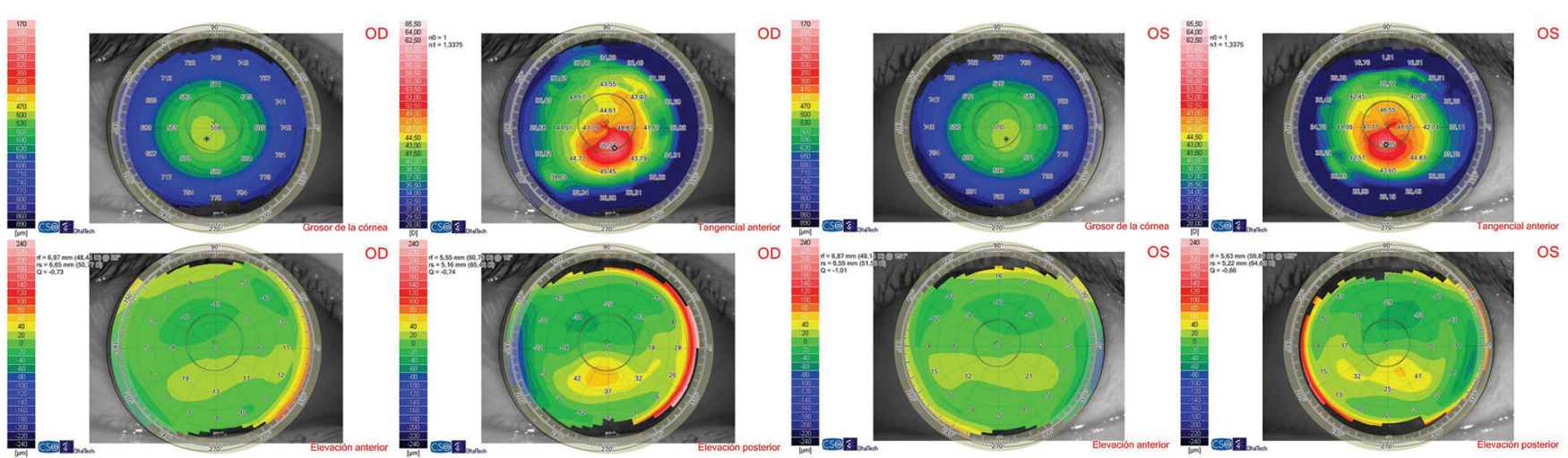

Fig. 1: Corneal analysis with the Sirius system in the right (four maps on the left) and left eyes (four maps on the right) of the case reported. Each analysis consisted of four maps displaying different type of information from the cornea analyzed: Pachymetric map (top left), tangential topographic map (top right), anterior elevation map (down left), and posterior elevation map (down right)
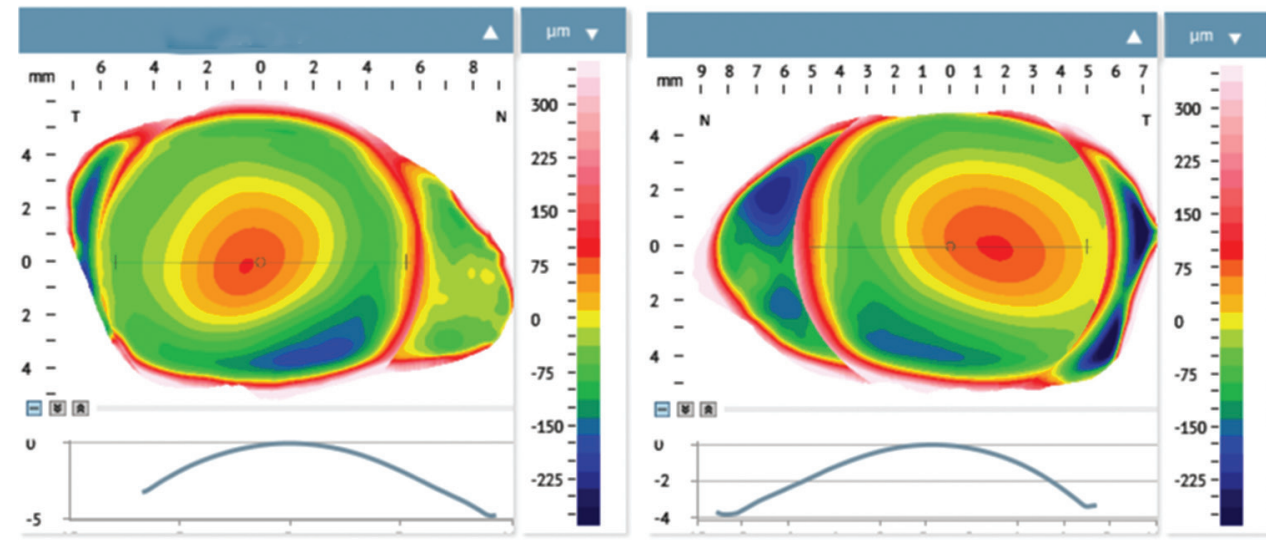

Fig. 2: Bisphere elevation map characterizing the corneo-scleral topographic profile (left, right eye; right, left eye)

touch: Central clearance zone (CCZ), peripheral central clearance zone (PCCZ), limbal clearance zone (LCZ), and scleral landing zone (SLZ). The lens is made of thermoset fluorosilicone acrylate copolymer derived primarily from siloxane acrylate, trifluoroethyl methacrylate, and methylmethacrylate with a water content of less than $1 \%$ (Paflufocon D) and Dk (oxygen permeability) of 100 Fatt (HDS 100 material, Paragon Vision Sciences, USA).

We initiated the fitting following the manufacturer's guidelines: Estimation of the sagittal height of the trial lens by adding $2000 \mu \mathrm{m}$ plus $300 \mu \mathrm{m}$ of apical clearance to the 10-mm corneal sagittal height measured with the swept source OCT system, DRI Triton from Topcon. According to this, we estimated a trial lens sagittal height of $4400 \mu \mathrm{m}$. Initially, we did not perform the fitting using sclera-corneal topographic data as no profilometer was available in our clinical setting. After the trial, the following CLs were fitted: ICD16.5/4400/-6.00 in RE and ICD16.5/4400/-10.00 in LE, achieving CDVA values of 0.00 and $0.10 \log$ MAR respectively. Mean apical clearance measured by OCT was $350 \mu \mathrm{m}$ in both eyes, with some level of inferior decentration and no visible peripheral scleral whitening. At 2 weeks of CL wear, the patient attended to our consultation with complaints of poor vision. An over-refraction of $-3.00-1.25 \times 140^{\circ}$ and $+1.00-1.00 \times 160^{\circ}$ was found in RE and LE respectively, with a significant level of inferior decentration but not abnormal scleral indentation. We suspect that a potential asymmetry of the sclero-conjunctival surface was inducing the positional instability of the lens with consequent generation of an irregular tear meniscus.

We decided to perform a refitting (after 2 weeks of discontinuation of $\mathrm{CL}$ ), but using the data obtained with a profilometer (ESP, Eaglet Eye b.v., Houten, The Netherlands). This device provided a complete analysis of the sclero-corneal topographic profile (Fig. 2). ${ }^{6}$ The bisphere map showed a clear asymmetry of the anterior scleral geometry in both eyes, with a less elevated nasal portion. Specifically, we found a difference between the nasal and temporal sagittal heights of 470 and $170 \mu \mathrm{m}$ in RE and LE respectively. This confirms the need for a peritoric scleral lens.

We decided to fit the toric version of the ICD scleral lens, with a toricity in the peripheral LCZ area of $5^{\circ}$ in the steep meridian (sagittal increase of $125 \mu \mathrm{m}$ in this meridian): ICDtoric16.5/4200/-4.75 $-1.25 \times 140^{\circ}$ and 


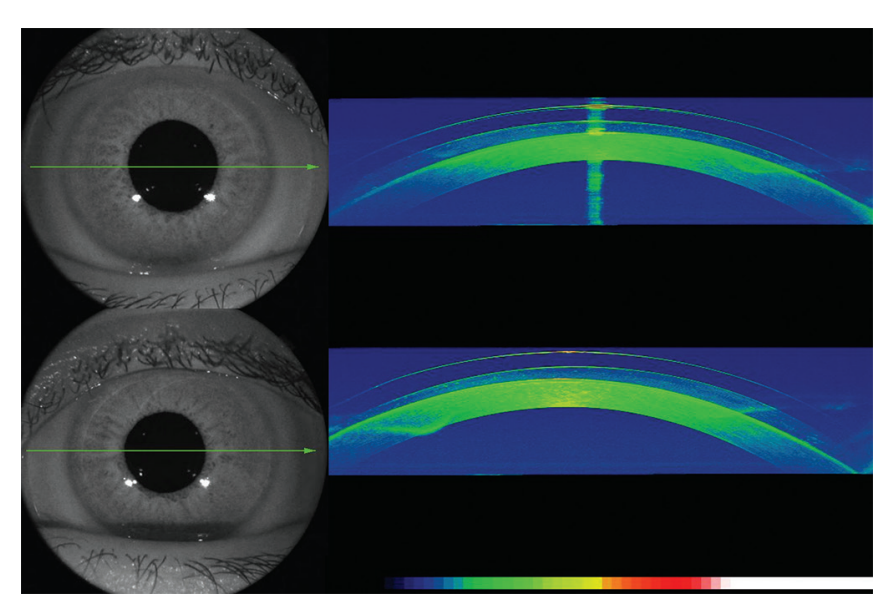

Fig. 3: Optical coherence tomography analysis of the final scleral lenses fitted in right (up) and left eyes (down) (left: Frontal image of the eye; right: Horizontal OCT scan showing the position of the lens)

ICDtoric16.5/4200/- $4.75-1.75 \times 60^{\circ}$ in RE and LE respectively. In both eyes, SLZ height was increased by $2^{\circ}$ (decrease of the sagittal height of the whole lens of around $50 \mu \mathrm{m}$ ) in order to solve an excess of peripheral bearing of the lens that was generating some level of scleral whitening. As expected according to the ESP measurements, this toricity was not enough to stabilize the lens and an additional increase of the peripheral toricity was required (LCZ $8^{\circ}$ steep meridian, an increase of approximately 200 $\mu \mathrm{m}$ in the sagittal height of the steep meridian). Therefore, the final lenses fitted were:

$$
\begin{aligned}
& \text { RE: ICD16.5 toric 4200/-4.75 -1.25 × 140\%SLZ-2/LCZ } \\
& +4 \text { steep } 8 \\
& \text { LE: ICD16.5 toric 4200/-4.75 -1.75 × 60\% /SLZ-2/LCZ } \\
& +4 \text { steep } 8
\end{aligned}
$$

With these two lenses, CL wear was comfortable and excellent visual acuity was achieved (CDVA RE: 0.00 $\log$ MAR, LE: $0.10 \log$ MAR). Mean apical clearance was 210 and $224 \mu \mathrm{m}$ in RE and LE respectively (Fig. 3). One year after this refitting, the visual performance with the CLs and comfortability remain intact.

\section{DISCUSSION}

Currently, new imaging techniques allow an optimization of the fitting of fully scleral CL, minimizing the number of trials required to obtain the most adequate fit. Besides OCT that allows the clinician to characterize the relationship among posterior CL surface and anterior corneal profiles, ${ }^{4,7}$ other novel techniques may be useful for the fitting of scleral lenses. We show in a specific case the usefulness of a corneo-scleral profilometry for obtaining a more optimized fitting of the peripheral zone of a fully scleral CL. This device provides a complete characterization of the corneal profile as well as of the geometry of the anterior sclera and limbus. ${ }^{8}$ To our knowledge, this is the first report of a scleral CL fitting guided or optimized by using the measurements provided by an eye surface profilometer.

We report the case of a patient with bilateral keratoconus fitted with a specific type of fully scleral CL following initially the manufacturer's guidelines and with no prefitting measurement of the sclero-conjunctival geometric profile. The ICD lens from Paragon was used, which has been shown in previous studies and series to be a good option for irregular corneas. ${ }^{9,10}$ We began the fitting with the spherical model of the lens, using a sagittal height calculated according to OCT measurements. The trial lens selected provided a comfortable wear and initially a good level of visual acuity, but the visual acuity worsened significantly at 15 days of wear. This suggested a positional instability of the CL leading to an irregular meniscus with a nonoptimized optical effect. For the type of CL used, when it is well-fitted, horizontal and vertical lens decentration has been demonstrated to follow an exponential decay over 8 hours that plateaus approximately at 2 hours after lens insertion, while central postlens tear layer thinning plateaus after 4 hours of lens wear. ${ }^{11}$ In our case, the behavior of the lens was different and potentially compatible with the high level of asymmetry of the scleral geometric profile.

After this, we performed a refitting using the toric model of the same scleral CL in order to check if better stability and visual performance were achieved. Simultaneously, we confirmed with the ESP system the presence of a high level of asymmetry of the anterior sclera, especially in the right eye, with more than $400 \mu \mathrm{m}$ of difference between the temporal and nasal sagittal heights. It is still unknown if this asymmetric scleral profile is common in keratoconus eyes as there are no studies reporting the distribution of the scleral geometry in this disease. However, differences in the shape of scleral limbus have been reported between normal healthy and keratoconus eyes. ${ }^{12}$ These differences may be the reason for the common need of toric scleral lenses when fitting advanced keratoconus cases. This should be investigated further in future studies. In our case, we tried to solve initially the case with a lens with a moderate level of peripheral toricity, following the recommendations of the manufacturer. However, this was not enough to stabilize the lens, as may be expected according to the measurements provided by the profilometer. Therefore, we decided to optimize the fit according to the scleral topographic measurements and to select a higher level of peripheral toricity. This provided a stabilization of the lens and an excellent visual performance. In a case like this, the use of the profilometer as a prefitting test would have allowed to optimize the procedure, avoiding the different trials that were required. 
In conclusion, fully scleral CL fitting may be optimized with the use of a profilometer, allowing the CL practitioner to select the most adequate design of the peripheral area of the lens and consequently allowing a better control of the peripheral bearing and stability of the lens. The potential benefit of corneo-scleral topographic devices for scleral CL fitting must be investigated further in future studies including large samples of eyes. Likewise, more studies are still needed providing more information about the geometry of the sclera in cases of irregular cornea, such as in keratoconus, in order to improve the fitting nomograms of fully scleral CLs in such cases.

\section{REFERENCES}

1. Parminder A, Jacobs DS. Advances in scleral lenses for refractive surgery complications. Curr Opin Ophthalmol 2015 Jul;26(4):243-248.

2. Downie LE, Lindsay RG. Contact lens management of keratoconus. Clin Exp Optom 2015 Jul;98(4):299-311.

3. Walker MK, Bergmanson JP, Miller WL, Marsack JD, Johnson LA. Complications and fitting challenges associated with scleral contact lenses: a review. Cont Lens Anterior Eye 2016 Apr;39(2):88-96.

4. Rathi VM, Mandathara PS, Dumpati S, Sangwan VS. Change in vault during scleral lens trials assessed with anterior segment optical coherence tomography. Cont Lens Anterior Eye 2017 Jun;40(3):157-161.
5. Consejo A, Llorens-Quintana C, Radhakrishnan H, Iskander DR. Mean shape of the human limbus. J Cataract Refract Surg 2017 May;43(5):667-672.

6. Jesus DA, Kedzia R, Iskander DR. Precise measurement of scleral radius using anterior eye profilometry. Cont Lens Anterior Eye 2017 Feb;40(1):47-52.

7. Esen F, Toker E. Influence of apical clearance on mini-scleral lens settling, clinical performance, and corneal thickness changes. Eye Contact Lens 2017 Jul;43(4):230-235.

8. Iskander DR, Wachel P, Simpson PN, Consejo A, Jesus DA. Principles of operation, accuracy and precision of an Eye Surface Profiler. Ophthalmic Physiol Opt 2016 May;36(3): 266-278.

9. Suarez C, Madariaga V, Lepage B, Malecaze M, Fournié P, Soler V, Galiacy S, Mély R, Cassagne M, Malecaze F. First experience with the ICD 16.5 mini-scleral lens for optic and therapeutic purposes. Eye Contact Lens 2018 Jan;44(1): 44-49.

10. Piñero DP. Fitting of a new design of full scleral contact lens in advanced keratoconus with previous implantation of intracorneal ring segments. Int J Kerat Ect Cor Dis 2015 May-Aug;4(2):56-59.

11. Vincent SJ, Alonso-Caneiro D, Collins MJ. The temporal dynamics of miniscleral contact lenses: central corneal clearance and centration. Cont Lens Anterior Eye 2017 Jul; pii:S1367-0484(17)30171-6.

12. Mas-Aixala E, Gispets J, Lupón N, Cardona G. The variability of corneal and anterior segment parameters in keratoconus. Cont Lens Anterior Eye 2016 Dec;39(6):466-470. 